$$
\begin{aligned}
& 7 N+10 \\
& 089435
\end{aligned}
$$

\title{
Western blot of stained proteins from dried polyacrylamide gels
}

\author{
Claudia Gruber and Helga Stan-Lotter1)
}

Institute of Genetics and General Biology, University of Salzburg, Hellbrunnerstr. 34, A-5020 Salzburg, Austria

Running title: Western blot from dried gels

Category: Immunological Procedures/Notes and Tips 
Western blotting of proteins is customarily performed following their separation on polyacrylamide gels, either prior to staining (1) or, as recently reported, following staining (2). We describe here Western blotting with stained gels, which had been dried and some of which had been stored for years. This procedure permits immunological analysis of proteins, to which antisera may have become available only later, or where the application of newly developed sensitive detection methods is desired. Once rehydration of the gels is achieved, proteins can be.transferred to blotting membranes by any appropriate protocol. Proteins stained with Coomassie Blue have to be detected with a non-chromogenic method, such as the film-based enhanced chemiluminescence $(E C L)^{2)}$ procedure (3). Silver stained proteins, which transfer in the colorless form, may be visualized by any detection method, although, because of the usually very low amounts of proteins, detection by ECL is preferable. Blotting of stained proteins from rehydrated gels is as rapid and as quantitative as from freshly prepared gels, in contrast to blotting from wet stained gels, which requires extensive washing and results in low transfer efficiency (2). Together with a photographic record of the gel pattern, unambiguous identification of immunoreactive proteins from complex mixtures is possible. Some further applications of this work are discussed.

\section{MATERIAL AND METHODS}

ATPases were prepared from the membranes of Halobacterium saccharovorum ATCC 29252 and Escherichia coli LE 392, respectively, as described previously (4). The ATPase from Thermus scotoductus DSM 8583 was prepared by a modification of the method of Yokoyama et al. (5). ATPase enzyme complexes consist of several subunits; most prominent are the two major subunits, which are designated $A$ and $B$ (H. saccharovorum) or $\alpha$ and $\beta$ (E.coli, T. scotoductus), respectively. Their apparent molecular masses are: $\mathrm{A}, 85 \mathrm{kDa} ; \mathrm{B}, 60 \mathrm{kDa} ; \alpha, 55 \mathrm{kDa}, \beta, 50 \mathrm{kDa}$. The minor subunits 
of the ATPase from E.coli are termed $\gamma, \delta$ and $\varepsilon$, with molecular masses of $32 \mathrm{kDa}$, $19 \mathrm{kDa}$ and $15 \mathrm{kDa}$, respectively. Antisera were raised in rabbits against the holoenzyme of $E$. coli (6) and against subunits $A$ and $B$ from $H$. saccharovorum (Stan-Lotter, unpublished). Separation of enzyme subunits was performed by polyacrylamide gel electrophoresis according to (7) or (8); gel thickness was 0.75 or $1 \mathrm{~mm}$. Proteins were stained with $0.15 \%$ Coomassie Blue in $25 \%$ isopropanol, $10 \%$ acetic acid. Alternatively, staining with silver was performed with the silver stain kit from BioRad. Following soaking in $2 \%$ glycerol, $10 \%$ acetic acid, gels were dried at $80^{\circ} \mathrm{C}$ with a vacuum gel dryer (BioRad) on Whatman filter paper no. 3 , or on cellophane (BioRad). Dried gels were rehydrated by submerging them in $4 \%$ methanol or distilled water. Gels peeled off the filter paper within a few seconds; from cellophane, gels were separated by gentle mechanical agitation. The gels were then placed in blotting buffer according to (1) or (9); following two changes of buffer. complete rehydration and equilibration was achieved within about $1 \mathrm{~h}$. Residual filter paper fibers on the gels did not interfere with transfer of proteins. The gels were placed upside down on a PVDF') (BioRad) or nitrocellulose (Millipore) membrane. Blotting conditions for minigels were $170-300 \mathrm{~mA}$ in a TransBlot chamber (BioRad) for 30 to $90 \mathrm{~min}$. Nonspecific binding sites were blocked with BlockerTM BLOTTO (nonfat dry milk in a Tris buffer; from Pierce) for $1 \mathrm{~h}$. Following incubation of blots with the primary antibody (dilution 1:100 in BLOTTO buffer) overnight, they were washed four times with Tris-buffered saline (100 mM Tris, $0.9 \% \mathrm{NaCl}, \mathrm{pH} 7.5)$, containing $0.1 \%$ Tween 20, and once with the same buffer, but omitting Tween 20 . Blots were then incubated with a goat anti-rabbit IgG-horse radish peroxidase conjugate (Pierce), which was diluted 1:20000 with BLOTTO buffer. One h later, the blots were washed again as described above and incubated with SuperSignal ${ }^{\mathrm{TM}}$ substrate (Pierce) for $5 \mathrm{~min}$. The SuperSignalTM substrate working solution was prepared just prior to use by mixing equal parts of Luminol Enhancer Solution and 
Stable Peroxide Solution (both from Pierce). Each blot was then placed in a plastic wrap, taking care to avoid drying; all air bubbles were removed, and the protected blot was exposed to X-ray film (Kodak) in a film cassette for $15 \mathrm{~s}$ up to several min. Optimal exposure time differed from blot to blot; before placing the blot against the film, it was rested for at least $5 \mathrm{~min}$ in order to prevent signal-overshoot. Protein patterns were recorded with electrophoresis duplicating paper (Kodak) according to the instructions of the manufacturer. Protein concentcations were determined by the method of Bradford (10).

\section{RESULTS AND DISCUSSION}

Bacterial ATPase subunits, which had been separated by SDS gel electrophoresis, stained with Coomassie Blue or silver, and subsequently dried under reduced pressure, were used for analysis on Western blots. The gels were rehydrated by placing them in water or $4 \%$ methanol; in the latter solvent, the original size of gels which contained $10-12 \%$ acrylamide, was retained. Small gels, e.g. minigels, were usually rehydrated without cracks, if the gels had not been damaged during drying. Even if some cracking occurred, gel pieces could be easily mounted together when preparing them for blotting (see Fig.1, panel I). The Coomassie Blue pattern following transfer to PVDF of the ATPase from $H$. saccharovorum is shown in Fig. 1 , panel I, lanes $1-4$. The immuno reactive bands were detected by ECL, following probing with antiserum against subunit A (Fig.1, panel II, lanes 1,2) or subunit B (Fig. 1, panel II, lanes 3,4). The shapes of stained protein bands simplify the localization of immuno reactive bands, or of spots, in the case of two-dimensional gels.

Fig. 2 shows a similar experiment as in Fig.1, using a silver stained gel (panel 1). The ATPase from E. coli (lane 4) and decreasing amounts of ATPase from $T$. scotoductus were applied (lanes 1-3). Following transfer to PVDF, the blot was probed with antiserum against purified ATPase from E.coli (Fig.2, panel II). All five 
subunits of the antigen ATPase reacted with the E.coli antiserum, whereas only the $\beta$ subunit of the ATPase of $T$. scotoductus was recognized by the antiserum. The $\beta$ subunits of F-ATPases contain the active site and are highly conserved between different species. Panel III of Fig.2 shows a "phantom" pattern, which consisted of metallic silver grains. Since the grains are uncharged, they did not migrate in the electric field during blotting, but stayed behind in the gel. They did not diffuse either; thus, only the (colorless) proteins were transferred to the PVDF mèmbrane. The observation that silver stain was not taken up by a blotting membrane had been reported already by Ranganathan and De (2). No protein was left in this gel after the transfer, since postblot staining with either Coomassie Blue or silver did not enhance the pattern. Less than $0.2 \mu \mathrm{g}$ of the smaller subunits of the E.coli ATPase were detected (Fig.2, panel II); this amount could be lowered at least by a factor of 20 , using less protein, but increased exposure times of blots treated with the ECL reagents (not shown).

We were able to confirm that long transfer times are necessary and that low transfer efficiency ensues, when stained wet gels are used for blotting, as has been pointed out previously (2). With dried, rehydrated gels, though, these problems were completely eliminated. This was likely due to an efficient removal of acetic acid and other volatile substances during gel drying, which is usually carried out at $80^{\circ} \mathrm{C}$ under reduced pressure. Thus, if a fast analysis of samples from stained gels is desired, it would even be advisable to include drying with a vacuum gel dryer (required time: about $1 \mathrm{~h}$ for $0.75 \mathrm{~mm}$ gels) and rehydrating ( $1 \mathrm{~h}$ or less), before performing Western blotting. Transfer times can be as short as 30 min with most gels, containing up to $12 \%$ acrylamide; we used also Tris-Tricine gels (8), with acrylamide concentrations of about $16.5 \%$, which required $2 \mathrm{~h}$ for efficient transfer (not shown).

$\mathrm{N}$-terminal sequencing by Edman-degradation of blotted bacterial ATPase subunits from dried and stained gels was not possible, presumably because $N$-termini 
became acetylated during the staining procedure. Although we did not attempt to solve this problem, it may be possible to avoid blockage by using alternative staining methods, omitting the acetic acid.

\section{CONCLUSIONS}

We show that electrophoretically separated proteins can be analysed by Western blotting, following rehydration of dried polyacrylamide gels. This procedure allows multiple uses of a single polyacrylamide gel electrophoresis run: after recording the protein pattern of the Coomassie Blue or silver stained gel, the dried gel may be stored for extended periods, until antibodies to proteins of interest become available, or until immuno detection of minute quantities of proteins is attempted with highly sensitive novel methods, as for instance chemiluminescence-based procedures combined with light enhancement (3). Rehydration of gels and subsequent blotting yields sharp patterns in the case of Coomassie Blue stained gels, whereas silver stained proteins transfer in the colorless form, but leave a phantom pattern in the spent gel. Immuno detection can be carried out with any customary detection system with blots from silver stained proteins; proteins stained with Coomassie Blue have to be detected by a non-chromogenic method, e.g. by ECL.

\section{ACKNOWLEDGMENT}

This work was supported by the Austrian Science Foundation (FWF), project $P 9144$ MOB, and by NASA Cooperative Agreement NCC 2-578, while HSL was a Principal Investigator with the SETI Institute. We thank Dr. L.I.Hochstein for a gift of $H$. saccharovorum, C. Radax for the preparation of ATPase from T. scotoductus and A. Mayer for a critical reading of the manuscript. 


\section{REFERENCES}

1. Gershoni, J.M., and Palade, G.E. (1983) Anal. Biochem. 131, 1-15.

2. Ranganathan, V., and De, P.K. (1996) Anal. Biochem. 234, 102-104.

3. Whitehead, T.P., Thorpe, G.H.G., Carter, T.J.N., Groucutt, C. and Kricka, L.J. (1983) Nature 305, 158-159.

4. Stan-Lotter, H., and Hochstein, L.I. (1989) Eur. J. Biochem. 179, 155-160.

5. Yokoyama, K., Oshima, T., and Yoshida, M. (1990) J. Biol. Chem. 265, 2194621950 .

6. Loo, T.W., Stan-Lotter, H., MacKenzie, D., Molday, R.S. , and Bragg, P.D. (1983) Biochim. Biophys. Acta 733, 274-282.

7. Laemmli, U.K. (1970) Nature (London) 227, 680-685.

8. Schägger, H., and von Jagow, G. (1987) Anal Biochem 166, 368-379.

9. Dunn, S.D. (1986) Anal. Biochem. 157, 144-153.

10. Bradford, M.M. (1976) Anal. Biochem. 72, 248-254. 
Footnotes:

1) To whom correspondence should be addressed. FAX 43-662-8044-144; e-mail: helga.stan-lotter@sbg.ac.at

2) Abbreviations used: ECL, enhanced chemiluminescence; PVDF, polyvinylidene difluoride 


\section{LEGENDS TO FIGURES}

Fig.1. Western blot of ATPase subunits from a dried polyacrylamide gel following rehydration. The major subunits $A$ and $B$ of the ATPase from $H$. saccharovorum are indicated to the left. The gel system of Laemmli (7) was used for separation; acrylamide concentration was $12 \%$. Approximately 5 (lanes 1,4 ) or 10 (lanes 2,3$) \mu \mathrm{g}$ of protein were applied. Transfer time to PVDF membrane was $90^{\circ} \mathrm{min}$. Panel I, PVDF membrane with Coomassie Blue pattern, which had co-transferred with the proteins during blotting; panel II, films following probing of blot with antiserum against subunit $A$ (lanes 1,2 ) or subunit $B$ (lanes 3,4 ). Visualization of immuno reactions was performed with the enhanced chemiluminescence (ECL) reagents as described in Methods. Exposure time of blots was 10-20 s.

Fig.2. Western blot following silver staining. ATPase subunits from $T$. scotoductus (lanes 1-3) and E. coli (lane 4) were separated and immuno reactions visualized as described in the legend to Fig.1. Subunits $\alpha$ to $\varepsilon$ are indicated to the right. Lanes 1-4 contained 2.5, 1.2, 0.3 and $2.5 \mu \mathrm{g}$ of protein, respectively. Panel I, ATPase subunits following silver staining; panel II, blot following transfer of ATPase subunits to PVDF; antiserum raised against the ATPase enzyme from E. coli was used. Panel III, "phantom" pattern of metallic silver grains remaining in the gel following transfer of proteins to the PVDF membrane. 

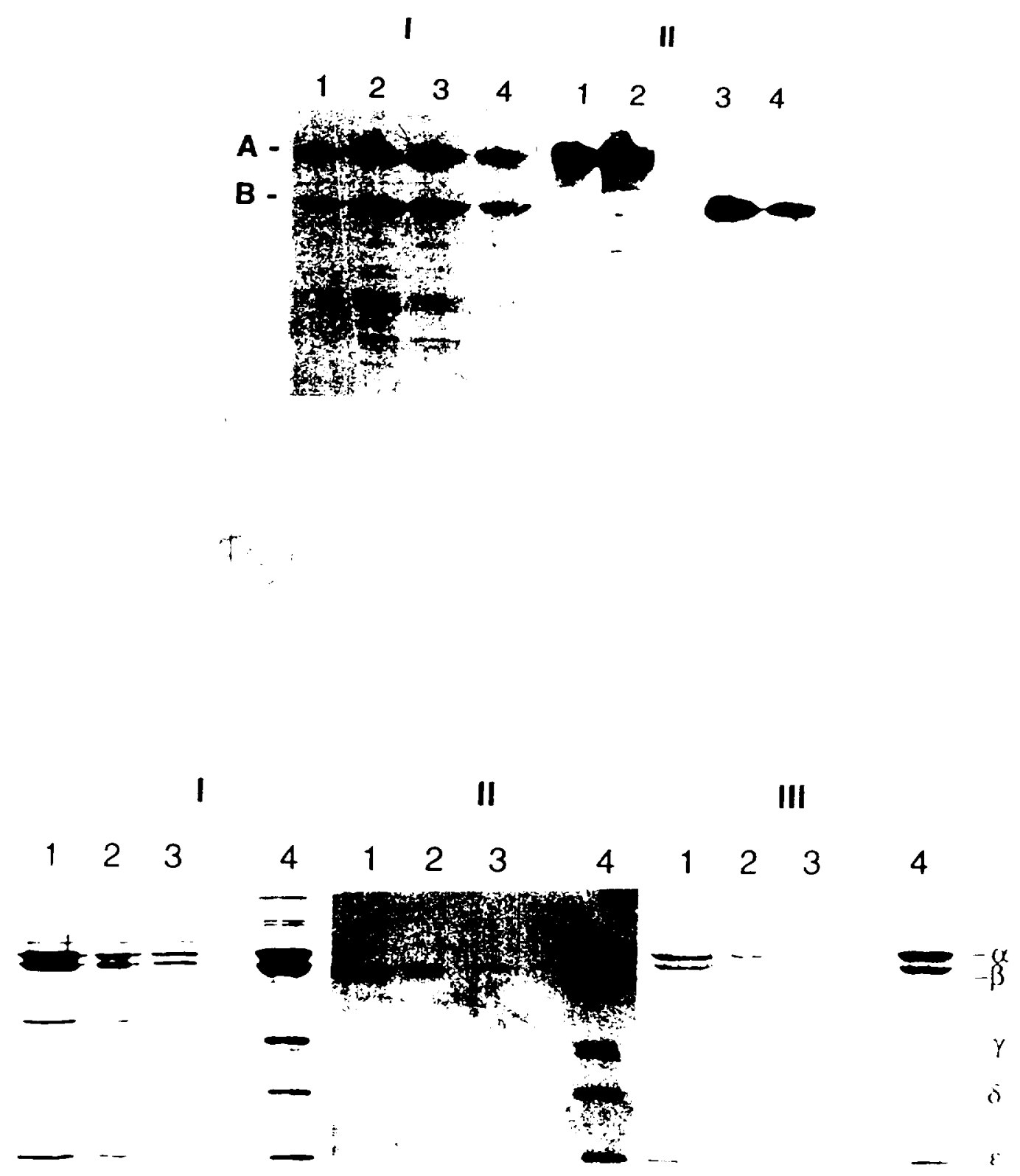